\title{
Extinction and Decay Estimates of Solutions for the $p$-Laplacian Equations with Nonlinear Absorptions and Nonlocal Sources
}

\author{
Xiulan Wu and Jun Fu \\ College of Mathematics, Jilin Normal University, Siping 136000, China \\ Correspondence should be addressed to Xiulan Wu; chjlsywxl@126.com
}

Received 18 April 2013; Accepted 19 July 2013

Academic Editor: Yanni Xiao

Copyright (C) 2013 X. Wu and J. Fu. This is an open access article distributed under the Creative Commons Attribution License, which permits unrestricted use, distribution, and reproduction in any medium, provided the original work is properly cited.

We investigate the extinction and decay estimates of the $p$-Laplacian equations with nonlinear absorptions and nonlocal sources. By Gagliardo-Nirenberg inequality, we obtain the sufficient conditions of extinction solutions, and we also give the precise decay estimates of the extinction solutions.

\section{Introduction}

In this paper, we consider the following fast diffusive $p$ Laplacian equation:

$$
\begin{gathered}
u_{t}=\operatorname{div}\left(|\nabla u|^{p-2} \nabla u\right)+\lambda \int_{\Omega} u^{q}(x, t) d x-k u^{r}, \\
x \in \Omega, t>0, \\
u(x, t)=0, \quad x \in \partial \Omega, t>0, \\
u(x, 0)=u_{0}(x), \quad x \in \Omega,
\end{gathered}
$$

where $1<p<2, k, q, \lambda>0,0<r<1, \Omega \subset R^{N}(N \geq 2)$ is a bounded domain with smooth boundary and $u_{0}(x) \in$ $L^{\infty}(\Omega) \cap W_{0}^{1, p}(\Omega)$ is a nonnegative function. Equation (1) is a class of nonlinear singular parabolic equations and appears to be relevant in the theory of non-Newtonian fluids perturbed by both nonlocal sources and nonlinear absorptions; see [14], for instance. Extinction is the phenomenon whereby the evolution of some nontrivial initial data $u_{0}(x)$ produces a nontrivial solution $u(x, t)$ in a time interval $0<t<T$ and $u(x, t) \rightarrow 0$ as $t \rightarrow T$. As an important property of solutions of developing equations, the extinction recently has been studied intensively by several authors in [5-9]. In paper [10], the authors discussed the extinction behavior of solutions for Problem (1)-(2) when $r=1$. In this paper, we investigated the extinction of solutions when $0<r<1$.
Due to the nature of our problem, we would like to use the following lemmas by [11].

Lemma 1 (Gagliardo-Nirenberg inequality). Suppose that $\beta \geq 0, N>p \geq 1, \beta+1 \leq q \leq(\beta+1) N p /(N-p)$; then for $u$ such that $|u|^{\beta} u \in W^{1, p}(\Omega)$, one has

$$
\|u\|_{q} \leq C^{1 /(\beta+1)}\|u\|_{r}^{1-\theta}\left\|\nabla\left(|u|^{\beta} u\right)\right\|_{p}^{\theta /(\beta+1)}
$$

with $\theta=\left((\beta+1) r^{-1}-q^{-1}\right) /\left(N^{-1}-p^{-1}+(\beta+1) r^{-1}\right)$, where $C$ is a constant depending only on $N$, $p$, and $r$.

\section{Main Results and Proofs}

Theorem 2. Assume that $p-1=q$ with $r<1$; then the nonnegative nontrivial weak solution of Problem (1)-(2) vanishes in finite time for any non-negative initial data provided that $|\Omega|$ or $\lambda$ is sufficiently small.

(1) For the case $2 N /(N+2) \leq p<2$, one has

$$
\begin{gathered}
\|u(\cdot, t)\|_{2} \leq\left(\left\|u_{0}\right\|_{2}^{2-k_{1}}-M_{1}\left(2-k_{1}\right) t\right)^{1 /\left(2-k_{1}\right)}, \\
t \in\left[0, T_{1}\right), \\
\|u(\cdot, t)\|_{2} \equiv 0, \quad t \in\left[T_{1},+\infty\right),
\end{gathered}
$$

where $k_{1}, M_{1}$, and $T_{1}$ are given by (11), (16), and (17), respectively. 
(2) For the case $1<p<2 N /(N+2)$, one has

$$
\begin{gathered}
\|u(\cdot, t)\|_{1+s} \leq\left(\left\|u_{0}\right\|_{1+s}^{1+s-k_{2}}-M_{2}\left(1+s-k_{2}\right) t\right)^{1 /\left(1+s-k_{2}\right)}, \\
t \in\left[0, T_{2}\right), \\
\|u(\cdot, t)\|_{2} \equiv 0, \quad t \in\left[T_{2},+\infty\right),
\end{gathered}
$$

where $s, k_{2}, M_{2}$, and $T_{2}$ are given by (18), (22), (26), and (28), respectively.

Proof. (1) For the case $2 N /(N+2) \leq p<2$, multiplying (1) by $u$ and integrating over $\Omega$, we deduce from the Hölder inequality that

$$
\frac{1}{2} \frac{d}{d t}\|u\|_{2}^{2}+\|\nabla u\|_{p}^{p}+k\|u\|_{r+1}^{r+1} \leq \lambda|\Omega|\|u\|_{p}^{p} .
$$

inequality

$$
\|u\|_{p} \leq B\|\nabla u\|_{p}
$$

where $B$ denotes the optimal embedding constant, combining (6) and (7) we have

$$
\frac{1}{2} \frac{d}{d t}\|u\|_{2}^{2}+\left(1-\lambda B^{p}|\Omega|\right)\|\nabla u\|_{p}^{p}+k\|u\|_{r+1}^{r+1} \leq 0 .
$$

By Lemma 1, we have

$$
\|u\|_{2} \leq C_{1}(N, p, r)\|\nabla u\|_{p}^{\theta}\|u\|_{1+r}^{1-\theta},
$$

where $\theta_{1}=(1 /(1+r)-1 / 2)(1 / N-1 / p+1 /(1+r))^{-1}$.

It is easy to check that $\theta_{1} \in(0,1]$; using Young's inequality with $\varepsilon$, it follows from (9) that

$$
\|u\|_{2}^{k_{1}} \leq C_{1}^{k_{1}}(N, p, r)\left(\varepsilon_{1}\|\nabla u\|_{p}^{p}+C\left(\varepsilon_{1}\right)\|u\|_{1+r}^{p k_{1}\left(1-\theta_{1}\right) /\left(p-k_{1} \theta_{1}\right)}\right),
$$

where $\varepsilon_{1}>0$ and $k_{1}>0$ will be determined later. We choose

$$
k_{1}=\frac{2[(1+r) p+N(p-1-r)]}{2 p+N(p-1-r)} .
$$

Then we can conclude that $k_{1} \in(1,2)$ and $p k_{1}\left(1-\theta_{1}\right) /(p-$ $\left.k_{1} \theta_{1}\right)=1+r$. Therefore, it follows from (10) that

$$
\|u\|_{1+r}^{1+r} \geq\left(C_{1}^{-k_{1}}(N, p, r)\|u\|_{2}^{k_{1}}-\varepsilon_{1}\|\nabla u\|_{p}^{p}\right) \frac{1}{C\left(\varepsilon_{1}\right)} .
$$

By combining (8) and (12), we have

$$
\begin{gathered}
\frac{1}{2} \frac{d}{d t}\|u\|_{2}^{2}+\left(1-\lambda B^{p}|\Omega|-\frac{k \varepsilon_{1}}{C\left(\varepsilon_{1}\right)}\right)\|\nabla u\|_{p}^{p} \\
+\frac{k C_{1}^{-k_{1}}(N, p, r)}{C\left(\varepsilon_{1}\right)}\|u\|_{2}^{k_{1}} \leq 0 .
\end{gathered}
$$

Choosing $\varepsilon_{1}$ small enough such that $1-k \varepsilon_{1} / C\left(\varepsilon_{1}\right)>0$ and $|\Omega| \leq\left(1-k \varepsilon_{1} / C\left(\varepsilon_{1}\right)\right) / \lambda B^{p}$, then we have $1-k \varepsilon_{1} / C\left(\varepsilon_{1}\right)-$ $B^{p} \lambda|\Omega|>0$. Therefore, we deduce from $k_{1} \in(1,2)$ that

$$
\frac{d}{d t}\|u\|_{2}+M_{1}\|u\|_{2}^{k_{1}-1} \leq 0
$$

which implies that

$$
\begin{gathered}
\|u(\cdot, t)\|_{2} \leq\left(\left\|u_{0}\right\|_{2}^{2-k_{1}}-M_{1}\left(2-k_{1}\right) t\right)^{1 /\left(2-k_{1}\right)}, \\
t \in\left[0, T_{1}\right), \\
\|u(\cdot, t)\|_{2} \equiv 0, \quad t \in\left[T_{1},+\infty\right),
\end{gathered}
$$

where

$$
\begin{gathered}
M_{1}=\frac{k C_{1}^{-k_{1}}(N, p, r)}{C\left(\varepsilon_{1}\right)}, \\
T_{1}=\frac{\left\|u_{0}\right\|_{2}^{2-k_{1}}}{M_{1}\left(2-k_{1}\right)} .
\end{gathered}
$$

(2) For the case $1<p<2 N /(N+2)$, multiplying (1) by $u^{s}$, where

$$
s>l=\frac{2 N-p(1+N)}{p}>1,
$$

integrating over $\Omega$, we deduce from the Hölder inequality that

$$
\begin{aligned}
& \frac{1}{1+s} \frac{d}{d t}\|u\|_{1+s}^{1+s}+\frac{s p^{p}}{(p+s-1)^{p}}\left\|\nabla u^{(p+s-1) / p}\right\|_{p}^{p} \\
& +k\|u\|_{s+r}^{s+r} \leq \lambda|\Omega|\|u\|_{p+s-1}^{p+s-1} .
\end{aligned}
$$

By Lemma 1 and $s>1$, we have

$$
\|u\|_{s+1} \leq C_{2}(N, p, r)\left\|\nabla u^{(p+s-1) / p}\right\|_{p}^{p \theta_{2} /(p+s-1)}\|u\|_{s+r}^{1-\theta_{2}},
$$

where $\theta_{2}=N(1-r)(p+s-1) /(s+1)[p(s+r)+N(p-1-r)]$. By (18) and $r<1$, it is easy to check that $\theta_{2} \in(0,1)$. By Young's inequality with $\varepsilon$, it follows from (19) that

$$
\begin{aligned}
\|u\|_{s+1}^{k_{2}} \leq C_{2}^{k_{2}}(N, p, r, s)\left(\varepsilon_{2}\right. & \left\|\nabla u^{(p+s-1) / p}\right\|_{p}^{p}+C\left(\varepsilon_{2}\right) \\
& \left.\times\|u\|_{s+r}^{\left(1-\theta_{2}\right) k_{2}(p+s-1) /\left(p+s-1-k_{2} \theta_{2}\right)}\right),
\end{aligned}
$$

where $\varepsilon_{2}>0$ and $k_{2}>0$ will be determined later. We choose

$$
k_{2}=\frac{(s+1)[(s+r) p+N(p-1-r)]}{(s+1) p+N(p-1-r)} ;
$$

then it follows that $k_{2} \in(s, s+1)$ and $(p+s-1) k_{2}\left(1-\theta_{2}\right) /(p+$ $\left.s-1-k_{2} \theta_{2}\right)=s+r$. Therefore, it follows from (21) that

$$
\|u\|_{s+r}^{s+r} \geq \frac{C_{2}^{-k_{2}}(N, p, r, s)}{C\left(\varepsilon_{2}\right)}\|u\|_{s+1}^{k_{2}}-\frac{\varepsilon_{2}}{C\left(\varepsilon_{2}\right)}\left\|\nabla u^{(p+s-1) / p}\right\|_{p}^{p} .
$$

By combining (19) and (23), we have by poincare inequality

$$
\begin{gathered}
\frac{1}{1+s} \frac{d}{d t}\|u\|_{1+s}^{1+s}+\left(\frac{s p^{p}}{(p+s-1)^{p}}-\frac{k \varepsilon_{2}}{C\left(\varepsilon_{2}\right)}-\lambda|\Omega| B^{p}\right) \\
\quad \times\left\|\nabla u^{(p+s-1) / p}\right\|_{p}^{p}+\frac{k C_{2}^{k_{2}}(N, p, r, s)}{C\left(\varepsilon_{2}\right)}\|u\|_{s+1}^{k_{2}} \leq 0 .
\end{gathered}
$$


Choosing $\varepsilon_{2}>0$ small enough such that $s p^{p} /(p+s-1)^{p}-$ $k \varepsilon_{2} / C\left(\varepsilon_{2}\right)>0$ and $|\Omega| \leq\left(s p^{p} /(p+s-1)^{p}-k \varepsilon_{2} / C\left(\varepsilon_{2}\right)\right) / \lambda|\Omega| B^{p}$, then we have $s p^{p} /(p+s-1)^{p}-k \varepsilon_{2} / C\left(\varepsilon_{2}\right)-\lambda|\Omega| B^{p}>0$. Therefore, we deduce from $k_{2} \in(s, s+1)$ that

$$
\frac{d}{d t}\|u\|_{1+s}+M_{2}\|u\|_{1+s}^{k_{2}-s} \leq 0
$$

where

$$
M_{2}=\frac{k C_{2}^{-k_{2}}(N, p, r, s)}{C\left(\varepsilon_{2}\right)},
$$

which implies that

$$
\begin{gathered}
\|u(\cdot, t)\|_{1+s} \leq\left[\left\|u_{0}\right\|_{1+s}^{1+s-k_{2}}-M_{2}\left(1+s-k_{2}\right) t\right]^{1 /\left(1+s-k_{2}\right)}, \\
t \in\left[0, T_{2}\right), \\
\|u(\cdot, t)\|_{1+s} \equiv 0, \quad t \in\left[T_{2},+\infty\right),
\end{gathered}
$$

where

$$
T_{2}=\frac{\left\|u_{0}\right\|_{1+s}^{1+s-k_{2}}}{M_{2}\left(1+s-k_{2}\right)} \text {. }
$$

The proof of Theorem 2 is complete.

Theorem 3. Assume that $r<1$.

(1) If $2 N /(N+2) \leq p<2$ with $q>k_{1}-1=(2 r p+$ $N(p-1-r)) /(2 p+N(p-1-r))$, then the non-negative nontrivial weak solution of Problem (1)-(2) vanishes in finite time provided that $u_{0}$ (or $|\Omega|$ or $\lambda$ ) is sufficiently small and

$$
\begin{gathered}
\|u(\cdot, t)\|_{2} \leq\left(\left\|u_{0}\right\|_{2}^{2-k_{1}}-\left(2-k_{1}\right) M_{3} t\right)^{1 /\left(2-k_{1}\right)}, \\
t \in\left[0, T_{3}\right), \\
\|u(\cdot, t)\|_{2} \equiv 0, \quad t \in\left[T_{3},+\infty\right)
\end{gathered}
$$

where $k_{1}, M_{3}$, and $T_{3}$ are given by (11), (35), and (33), respectively.

(2) If $1<p<2 N /(N+2)$ with $q>k_{2}-s=((s+1) r p+$ $N(p-1-r)) /((s+1) p+N(p-1-r))$, then the nonnegative nontrivial weak solution of Problem (1)-(2) vanishes iffinite time provided that $u_{0}($ or $|\Omega|$ or $\lambda)$ is sufficiently small and

$$
\begin{array}{r}
\|u(\cdot, t)\|_{s+1} \leq\left(\left\|u_{0}\right\|_{s+1}^{s+1-k_{2}}-\left(s+1-k_{2} M_{4}\right) t\right)^{1 /\left(s+1-k_{2}\right)}, \\
t \in\left[0, T_{4}\right),
\end{array}
$$$$
\|u(\cdot, t)\|_{s+1} \equiv 0, \quad t \in\left[T_{4},+\infty\right),
$$

where $s, k_{2}, M_{4}$, and $T_{4}$ are given by (18), (22), (39), and (41), respectively.
Proof. (1) If $2 N /(N+2) \leq p<2$, multiplying (1) by $u$ and integrating over $\Omega$, we deduce from (12) and the Hölder inequality that

$$
\begin{gathered}
\frac{1}{2} \frac{d}{d t}\|u\|_{2}^{2}+\left(1-\frac{k \varepsilon_{1}}{C\left(\varepsilon_{1}\right)}\right)\|\nabla u\|_{p}^{p}+\frac{k C_{1}^{-k_{1}}(N, p, r)}{C\left(\varepsilon_{1}\right)} \\
\times\|u\|_{2}^{k_{1}}-\lambda|\Omega|^{(3-q) / 2}\|u\|_{2}^{q+1} \leq 0 .
\end{gathered}
$$

By choosing $\varepsilon_{1}>0$ small enough such that $1-k \varepsilon_{1} / C\left(\varepsilon_{1}\right) \geq 0$, we obtain that

$$
\frac{d}{d t}\|u\|_{2}+M_{3}\|u\|_{2}^{k_{1}-1} \leq 0
$$

provided that $\left\|u_{0}\right\|_{2} \leq\left(k C_{1}^{-k_{1}}(N, p, r) / C\left(\varepsilon_{1}\right) \lambda|\Omega|^{(3-q) / 2}\right)^{1 /\left(q-k_{1}+1\right)}$ and $q>k_{1}-1=(2 r p+N(p-1-r)) /(2 p+N(p-1-r))$, where

$$
M_{3}=\frac{k C_{1}^{-k_{1}}(N, p, r)}{C\left(\varepsilon_{1}\right)}-\lambda|\Omega|^{(3-q) / 2}\left\|u_{0}\right\|_{2}^{q-k_{1}+1}>0 .
$$

From $(32)$ and $k_{1} \in(1,2)$, we can derive that

$$
\begin{gathered}
\|u(\cdot, t)\|_{2} \leq\left(\left\|u_{0}\right\|_{2}^{2-k_{1}}-\left(2-k_{1}\right) M_{3} t\right)^{1 /\left(2-k_{1}\right)}, \\
t \in\left[0, T_{3}\right), \\
\|u(\cdot, t)\|_{2} \equiv 0, \quad t \in\left[T_{3},+\infty\right),
\end{gathered}
$$

where

$$
T_{3}=\frac{\left\|u_{0}\right\|_{2}^{2-k_{1}}}{\left(2-k_{1}\right) M_{3}} .
$$

(2) If $1<p<2 N /(N+2)$, multiplying (1) by $u^{s}$, where $s$ is given by (18) and integrating over $\Omega$, we deduce from the Hölder inequality and (23) that

$$
\begin{gathered}
\frac{1}{1+s} \frac{d}{d t}\|u\|_{1+s}^{1+s}+\left(\frac{s p^{p}}{(p+s-1)^{p}}-\frac{k \varepsilon_{2}}{C\left(\varepsilon_{2}\right)}\right)\left\|\nabla u^{(p+s-1) / p}\right\|_{p}^{p} \\
+\frac{k C_{2}^{-k_{2}}(N, p, r, s)}{C\left(\varepsilon_{2}\right)}\|u\|_{s+1}^{k_{2}} \leq \lambda\|u\|_{1+s}^{q+s}|\Omega|^{(2+s-q) /(1+s)}
\end{gathered}
$$

Choosing $\varepsilon_{2}>0$ small enough such that $s p^{p} /(p+s-1)^{p}-$ $k \varepsilon_{2} / C\left(\varepsilon_{2}\right)>0$, we have

$$
\begin{aligned}
\frac{d}{d t}\|u\|_{1+s}+\|u\|_{1+s}^{k_{2}-s} & \left(\frac{k C_{2}^{k_{2}}(N, p, r, s)}{C\left(\varepsilon_{2}\right)}\right. \\
& \left.-\lambda|\Omega|^{(2+s-q) /(1+s)}\|u\|_{1+s}^{q+s-k_{2}}\right) \leq 0 .
\end{aligned}
$$

Therefore, we have

$$
\frac{d}{d t}\|u\|_{1+s}+M_{4}\|u\|_{1+s}^{k_{2}-s} \leq 0
$$


provided that $\left\|u_{0}\right\|_{1+s} \leq\left(k C_{2}^{-k_{2}}(N, p, r, s) / C\left(\varepsilon_{2}\right) \lambda\right.$ $\left.|\Omega|^{(2+s-q) /(1+s)}\right)^{1 /\left(q+s-k_{2}\right)}$ and $q>k_{2}-s=((s+1) r p$ $+N(p-1-r)) /((s+1) p+N(p-1-r))$, where

$$
M_{4}=\frac{k C_{2}^{-k_{2}}(N, p, r, s)}{C\left(\varepsilon_{2}\right)}-\lambda|\Omega|^{(2+s-q) /(1+s)}\left\|u_{0}\right\|_{1+s}^{q+s-k_{2}}>0 .
$$

It follows from (38) and $k_{2} \in(s, s+1)$ that

$$
\begin{gathered}
\|u(\cdot, t)\|_{1+s} \leq\left(\left\|u_{0}\right\|_{s+1}^{s+1-k_{2}}-M_{4}\left(s+1-k_{2}\right) t\right)^{1 /\left(s+1-k_{2}\right)}, \\
t \in\left[0, T_{4}\right), \\
\|u(\cdot, t)\|_{s+1} \equiv 0, \quad t \in\left[T_{4},+\infty\right),
\end{gathered}
$$

where

$$
T_{4}=\frac{\left\|u_{0}\right\|_{1+s}^{1+s-k_{2}}}{M_{4}\left(s+1-k_{2}\right)}
$$

The proof of Theorem 3 is complete.

\section{Acknowledgments}

This work is supported by the Department of Education of Jilin Province (2013445) and by Science and Technology Bureau of Siping, Jilin Province (2012040), and is partially supported by the NSF of China under Grant 11171060.

\section{References}

[1] Z. Wu, J. Zhao, J. Yin, and H. Li, Nonlinear Diffusion Equation, World Scientific, Singapore, 2001.

[2] E. DiBenedetto, Degenerate Parabolic Equations, Springer, Berlin, Germany, 1993.

[3] J. N. Zhao, "Existence and nonexistence of solutions for $u_{t}=$ $\operatorname{div}\left(|\nabla u|^{p-2} \nabla u\right)+f(\nabla u, u, x, t)$, , Journal of Mathematical Analysis and Applications, vol. 172, no. 1, pp. 130-146, 1993.

[4] N. D. Alikakos and L. C. Evans, "Continuity of the gradient for weak solutions of a degenerate parabolic equation," Journal de Mathématiques Pures et Appliquées, vol. 62, no. 3, pp. 253-268, 1983.

[5] W. Liu, "Extinction properties of solutions for a class of fast diffusive $p$-Laplacian equations," Nonlinear Analysis. Theory, Methods \& Applications A, vol. 74, no. 13, pp. 4520-4532, 2011.

[6] C. Mu, L. Wang, and P. Zheng, "Extinction and non-extinction for a polytropic filtration equation with absorption and source," Journal of Mathematical Analysis and Applications, vol. 391, no. 2, pp. 429-440, 2012.

[7] P. Zheng and C. Mu, "Extinction and decay estimates of solutions for a polytropic filtration equation with the nonlocal source and interior absorption," Mathematical Methods in the Applied Sciences, vol. 36, no. 6, pp. 730-743, 2013.

[8] B. Wu, "Global existence and extinction of weak solutions to a class of semiconductor equations with fast diffusion terms," Journal of Inequalities and Applications, vol. 2008, Article ID 961045, 14 pages, 2008.
[9] J. Yin, J. Li, and C. H. Jin, "Non-extinction and critical exponent for a polytropic filtration equation," Nonlinear Analysis. Theory, Methods \& Applications A, vol. 71, no. 1-2, pp. 347-357, 2009.

[10] Z. B. Fang and X. Xu, "Extinction behavior of solutions for the $p$-Laplacian equations with nonlocal sources," Nonlinear Analysis. Real World Applications, vol. 13, no. 4, pp. 1780-1789, 2012.

[11] C. Caisheng and W. Ruyun, " $L^{\infty}$ estimates of solution for the evolution $m$-Laplacian equation with initial value in $L^{q}(\Omega)$," Nonlinear Analysis. Theory, Methods \& Applications A, vol. 48, no. 4, pp. 607-616, 2002. 


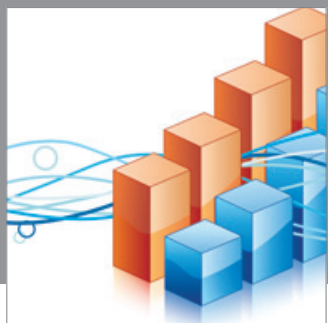

Advances in

Operations Research

mansans

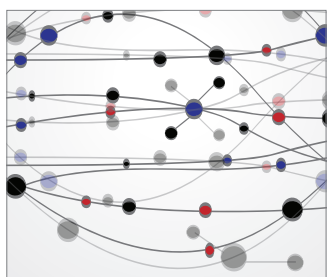

The Scientific World Journal
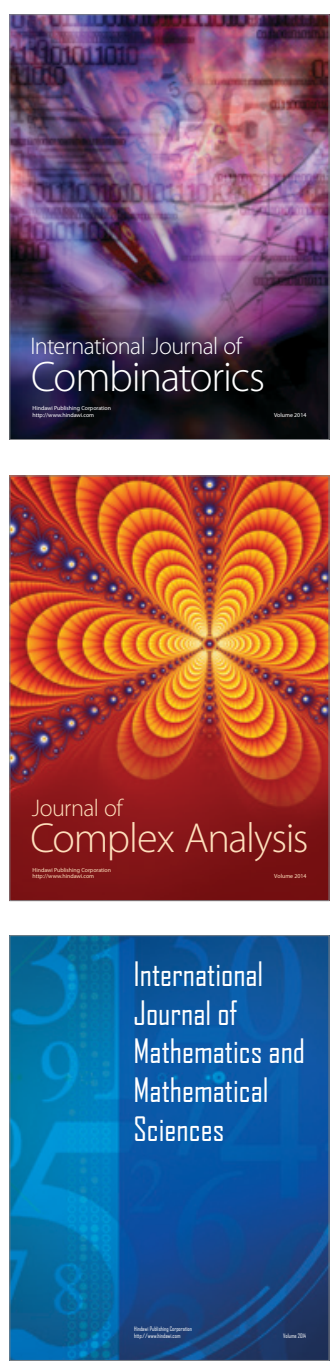
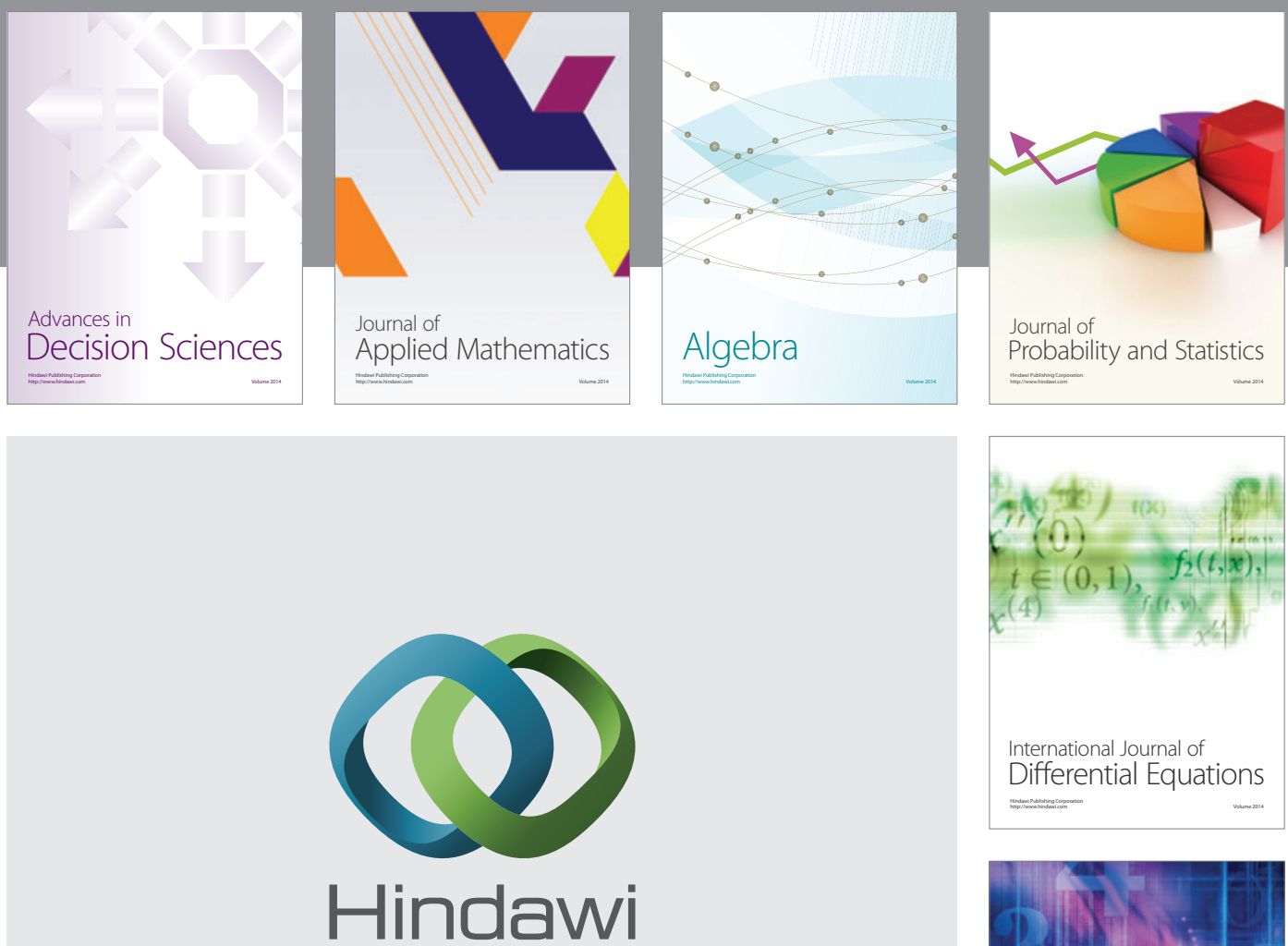

Submit your manuscripts at http://www.hindawi.com
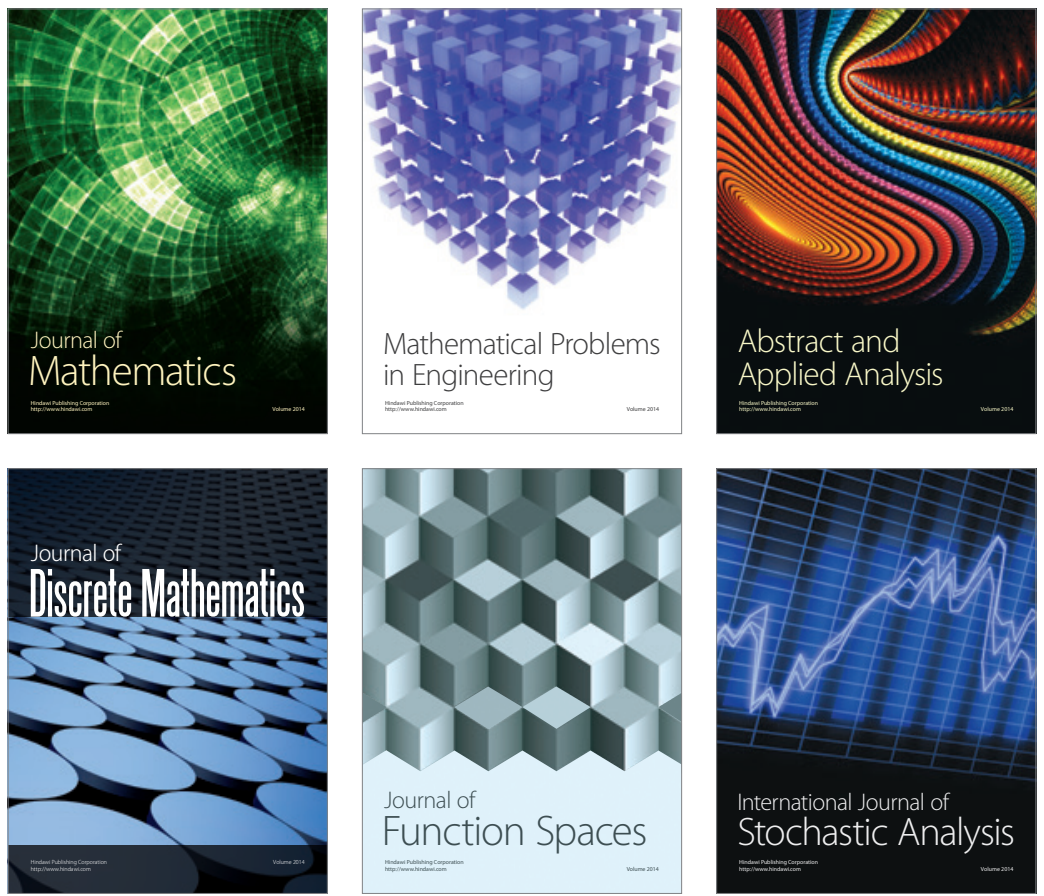

Journal of

Function Spaces

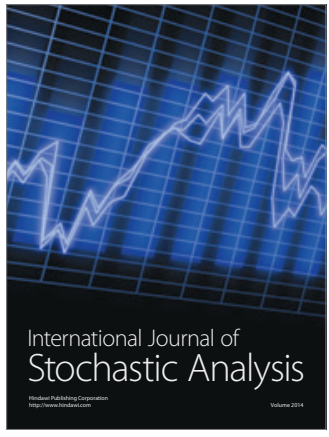

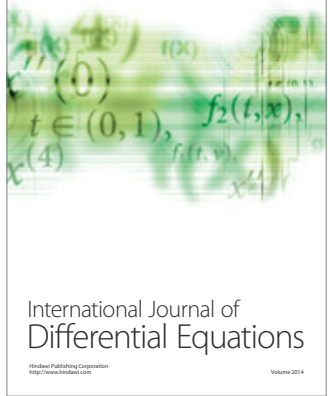
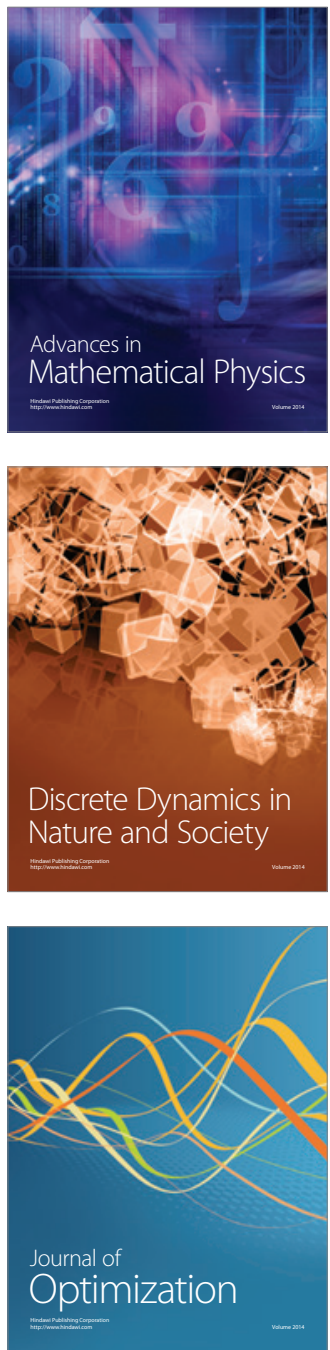